\title{
ARTICULO ORIGINAL-2016: Recuperación post- quirúrgica mejorada (fast-track) en el paciente adulto mayor
}

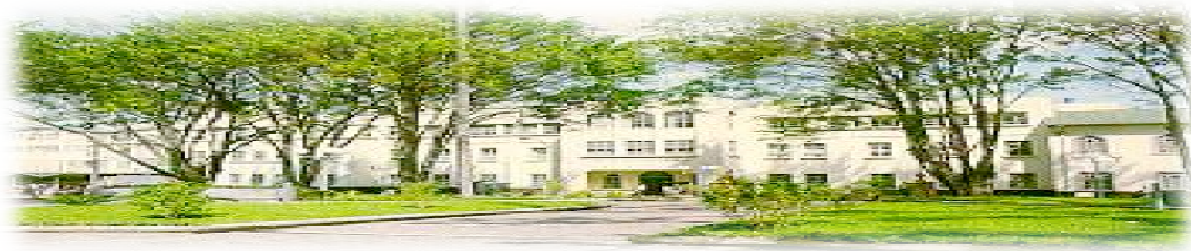

Hospital San Juan de Dios, San José, Costa Rica. Fundado en 1845
ISSN

Recibido: 19/10/2015

Aceptado: 21/11/2015

Araní Guánchez Mercado ${ }^{1}$

Gustavo Jiménez Ramirez ${ }^{2}$

Erika Cyrus Barker ${ }^{3}$

\begin{abstract}
${ }^{1}$ Licenciada en Medicina y Cirugía. Master en Rehabilitación Funcional del Adulto Mayor. Costa Rica.
${ }^{2}$ Especialista en Cirugía General y Laparoscopía avanzada. Profesor de pregrado y posgrado en la UCR y Universidad de Ciencias Médicas. Médico asistente especialista en cirugía general en Hospital México. Costa Rica.

${ }^{3}$ Doctorado en Rehabilitación Física. Directora de carrera en la Universidad Santa Paula (USP). Profesora de posgrado y pregrado de Rehabilitación Física en la USP. Costa Rica
\end{abstract}

\section{RESUMEN}

En los últimos años se ha incrementado la frecuencia de intervenciones quirúrgicas en la población geriátrica y este está asociado a una morbimortalidad alta. Ante esta realidad, indicadores validados y protocolos para el cuido especializado pre, trans y post quirúrgico del paciente adulto mayor (PAM) están siendo desarrollados para mejorar los resultados de las intervenciones. Los PAM poseen características particulares que los hacen uno de los grupos etáreos de salud más delicados, razón por la cual la atención de sus afecciones deben ser de abordaje multidisciplinarios y la prevención debe jugar un papel fundamental.El protocolo de tratamiento conocido como fast track (FT, por sus siglas en inglés), también conocido como recuperación postquirúrgica mejorada "ERAS" (según sus siglas en inglés: Enhanced Recovery After Surgery), es seguido con el fin de mejorar los resultados de las intervencionesen el paciente quirúrgico en los periodos pre-quirúrgico, transquirúrgico y post-quirúrgico.

Mediante esta revisión se pretende repasar el tema del protocolo ERAS de un punto de vista fisiológico y funcional y además, destacar porque se puede aplicar de manera segura y ventajosa al PAM. Adicionalmente, repasar las características del PAM y las necesidades de atención especializada de valoración y seguimiento como paciente quirúrgico.

\section{PALABRAS CLAVE}

Fast-Track, ERAS, rehabilitación funcional, paciente adulto mayor, cirugía, recuperación postquirúrgica mejorada. 


\section{ABSTRACT}

In the recent years there has been arise in the amount of surgical interventions in the elderly population. Given this reality, valid indicators and protocols for the specialized care in the pre, trans and post-surgical stages of the elderly patient are being developed to improve the outcomes and decrease de morbidity of the surgical interventions. The elderly patient tends to have complex health problems, reason why they should be attended in a multidisciplinary manner with prevention playing a major role. The protocol known as fast track (FT) or enhanced recovery after surgery (ERAS) is applied with the purpose to decrease the final outcome of the interventions in the surgical patient.

The objective of this review is to assess the ERAS protocol from a physiological and functional point of view and distinguish why it can be applied to the elderly patient in a safe and beneficial manner. Additionally, a review of the special attention requirements and follow-ups needed as an elderly patient undergoing surgery.

\section{KEY WORDS}

Fast-Track, functional rehabilitation, elderly patient, surgery, early rehabilitation after surgery.

\section{INTRODUCCIÓN}

Durante los últimos siglos la humanidad ha sufrido un cambio demográfico impulsado por un aumento de más de un $50 \%$ de la expectativa de vida, el cual conlleva a un aumento en la proporción de la población mayor de 65 años comúnmente referida como "adultos mayores" ${ }^{(1)}$.Esta situación ha impactado y cambiado la dinámica de los campos sociales, económicos y médicos, por ejemplo, en Costa Rica se estimó que en el año 2000 el $4.5 \%$ de la población era mayor de 65 años, y que para el 2030 se espera un aumento de un $8 \%$, y para el 2050 un $13.8 \%{ }^{(1)}$.

La población geriátrica representa una población que se caracteriza por ser pacientes delicados con una reserva fisiológica disminuida además de tener con frecuencia patologías crónicas y comorbilidades asociadas. Tales condiciones los hacen vulnerable a situaciones de estrés fisioló- gico, como es la cirugía. Ante esta situación, se hace evidente la necesidad de realizar estudios que exploren la efectividad de los protocolos de tratamiento para optimizar la recuperación posquirúrgica del PAM y, consecuentemente, reducir las posibilidades de complicaciones y lograr un egreso del hospital más expedito y seguro del paciente.

El protocolo de rehabilitación Fast Track o ERAS fue introducido por Kehlet en el año 2001 (2), con el fin de cambiar el cuido convencional (CC) de pacientes sometidos a cirugías colorrectales, para lograr un impacto positivo en la recuperación al paciente ${ }^{(3-4)}$. Se trata de un abordaje multimodal que incluye una serie de procedimientos e intervenciones con el fin de mejorar el manejo global del paciente, disminuir el estrés, las complicaciones, acortar el tiempo de la estadía hospitalaria y con ello reducir el costo total de la hospitalización. Según Spanjersberg WR et al en una revisión sistemática realizada de 17 estudios se concluyó que al aplicar el protocolo ERAS en la cirugía colorrectal, no hubo aumento en la mortalidad ni en la readmisión a 30 días pero sí una disminución de las complicaciones y estancia hospitalaria en comparación el $\mathrm{CC}^{(5)}$.

Posterior al éxito del protocolo ERAS en la cirugía colorrectal, se ha aplicado en otras cirugías siempre mostrando un impacto positivo en la recuperación del paciente. Según Aditya $\mathrm{J}$ et al en un estudio prospectivo intervencional incluyendo 60 sujetos sometidos a cirugías gastrointestinales varias, hubo una mejoría de 7.27 (1.36) días en la estancia hospitalaria sin empeoro en la readmisión o complicaciones ${ }^{(6)}$. Aún con la seguridad y ventaja establecida del protocolo aún no se dispone de suficientes evidencias de su efectividad

en cuanto a su aplicación en una población etárea específica, como es la población geriátrica.

\section{DISCUSIÓN}

\section{El protocolo "Fast track" o ERAS}

El protocolo ERAS involucra componentes que abarcan las diferentes etapas quirúrgicas que en conjunto contribuyen con la recuperación más expedita del paciente. Entre ellas se tienen las siguientes: (i) la educación sobre el protocolo al 
paciente o cuidador/familia, (ii) no preparación intestinal, (iii) carga de carbohidratos $2 \mathrm{hrs}$ previos a la cirugía, (iv) uso conservador de sondas, vías venosas/arteriales y drenajes, (v) restricción de líquidos trans-quirúrgicos, (vi) alimentación oral, (vii) deambulación temprana postoperatoria y (viii) el egreso temprano ${ }^{(7-8)}$. (Cuadro I). Cada componente del protocolo discutido aborda una vía fisiopatológica que se altera durante la cirugía con el fin de reducir el estrés quirúrgico y ayudar la recuperación integrada y expedita del paciente. Es importante notar que cada protocolo se ajusta de acuerdo a la cirugía en cuestión, por ejemplo según Kehlet $\mathrm{H}$ et al, el tipo de anestesia debe ser escogida tomando en cuenta las comorbilidades pre-existentes, el tipo de cirugía y el funcionamiento orgánico del paciente $^{(3)}$. Dado a su carácter multimodal, este protocolo se debe llevar a cabo a través de un equipo multidisciplinario, integrado por nutricionistas, enfermeras, cirujanos y anestesiólogos ${ }^{(9)}$.

\section{Fisiopatología}

Durante y después de la cirugía, el cuerpo responde con cambios a nivel del sistema neurológico, endocrino y metabólico, y del funcionamiento normal de los órganos ${ }^{(10)}$. Estos cambios generalmente son los siguientes: (i) aumento de hormonas catabólicas y disminución de las anabólicas, asociado a un hipermetabolismo; (ii) aumento del estímulo autonómico y aumento de trabajo cardiaco; (ii) función pulmonar debilitada; (iv) alteraciones gastrointestinales; (v) cambio en el sistema fibrinolítico de la coagulación, favoreciéndola; (vi) pérdida de la masa muscular e (vii) la inmunosupresión ${ }^{(3,10)}$.

El éxito de este protocolo es fundamentado en la atenuación del estrés peri-operatorio ${ }^{(11-13)}$. Sin embargo, también se sugiere que sus beneficios son mediados por el retorno más rápido del funcionamiento normal de los órganos ${ }^{(14)}$.

\section{El paciente adulto mayor quirúrgico}

La causa más frecuente de intervención quirúrgica en el PAM es el cáncer, la cual es una enfermedad primaria asociada al envejecimiento ${ }^{(15)}$. Sin embargo, no se dispone de evidencias de que esta sea más agresiva con la edad, pero los atrasos en su diagnóstico son los que generalmente resultan en complicaciones, que llevan a la nece- sidad del tratamiento quirúrgico ${ }^{(16)}$. La asociación de cáncer con el envejecimiento ha sido explicada mediante tres mecanismos no excluyente entre ellos, como son: la duración de la carcinogénesis, el aumento de la susceptibilidad que experimentan los tejidos envejecidos y los factores o cambios ambientales que favorecen la carcinogénesis ${ }^{(17)}$.

La edad avanzada es independiente en la predicción de los resultados de cirugías ${ }^{(18)}$. Sin embargo, se sugiere que son factores fisiológicos, en vez de etáreos, los que pueden afectar los resultados del PAM sometidos a cirugía ${ }^{(19)}$. Al respecto, existen diferentes términos utilizados para describir los factores fisiológicos que afectan al PAM, como son: la calidad de vida, fragilidad, funcionalidad e independencia.

La calidad de vida es un aspecto relativamente difícil de evaluar en el PAM, esto debido a que las auto valoraciones pueden ser desacertadas en un $40 \%{ }^{(20)}$. Dado el caso, se usan diferentes escalas de valoración como son las actividades instrumentales de la vida diaria (AIVD) y actividades básicas de la vida diaria $(\mathrm{ABVD})^{(21)}$. (Cuadro II).

La fragilidad se considera un sinónimo de discapacidad y co-morbilidad, que con frecuencia coincide con la edad avanzada. La definición estandarizada se fundamenta en cinco características: (i) pérdida de peso no intencional, (ii) debilidad, (iii) cansancio y baja resistencia física, (iv) lentitud y (v) bajo nivel de actividad ${ }^{(19-21)}$.Se han utilizado diferentes índices para cuantificar la fragilidad, creados con el fin de la identificación del paciente geriátrico frágil y la cuantificación de esta (Cuadro III).

El estado funcional de un adulto mayor es estimado con base en su independencia y tres índices: (i) Índice de Barthel, (iii) Índice de Capacidad Funcional y (iii) Medida de Independencia Funcional, (Cuadro IV). La mayor parte de la literatura sobre la dependencia funcional del adulto mayor y cirugías está basada en el monitoreo de la rehabilitación de traumas o fracturas de cadera ${ }^{(22)}$.

Se ha confirmado que el PAM tiende a ser un paciente de manejo complejo especialmente al 
Cuadro I: Descripción de los componentes del protocolo ERAS ${ }^{(6-8)}$.

\section{Componentes del protocolo ERAS y su descripción}

Educación: Antes de la cirugía respectiva, se le explica al paciente y/o familiares sobre lo que es el protocolo ERAS, pidiéndole su colaboración y dándole respuesta a cualquier pregunta o duda que puedan tener. Adicionalmente, se debe diseñar y establecer una red de apoyo para la recuperación del paciente cuando el mismo esté de vuelta en su domicilio.

No preparación intestinal: Actualmente la preparación del intestino con enemas y ayunas prolongadas es rutinaria como parte del CC. En el caso de ERAS se omite esta preparación y la ayunas se reducen a horas antes de la intervención. Algunos autores sugieren que la preparación intestinal no pareciera generar mejores resultados quirúrgicos en cuanto la disminución de sepsis o fuga anastomótica. Incluso, hay autores que afirman que su aplicación en ciertos casos puede empeorar el estado general del PAM ${ }^{(7)}$.

Carga de carbohidratos: Se le suministra al paciente una bebida rica en carbohidratos unas 2-3 horas antes a la cirugía, con lo cual se ha notado una apreciable disminución de la sed y la ansiedad, así como un aumento del bienestar post-quirúrgico del paciente ${ }^{(6)}$.

Duración corta e analgesia epidural y bloqueos locales: En los casos que lo permite el tipo de cirugía y la condición del paciente se debe utilizar un bloqueo epidural continuado por máximo 48 horas. El equipo no debe intervenir con la movilización del paciente.

Uso conservador de sondas y drenajes: El uso rutinario de drenajes abdominales y sondas nasogástricas debe ser evitado en lo posible, puesto que no se dispone de pruebas suficientemente confiables de su beneficio y estas pueden causar dolor, dificultades de movimiento y retraso en la ambulación temprana.

Restricción de líquido trans-quirúrgico: Durante la cirugía se reduce al mínimo la cantidad de líquido intra-venoso (IV) suministrado, a fin de evitar en el paciente una sobrecarga de líquido con sus posibles consecuencias negativas.

Oxígeno de alta concentración: Durante la cirugía se recomienda que oxígeno al $80 \%$ debe ser administrado y después continuado hasta 6 horas postoperatorias.

Uso de analgésicos orales y no opiodes: En cuanto al suministro de analgésicos y anti-inflamatorios, se recomienda prescribir acetaminofén y anti-inflamatorios no esteroideos (AINES) preferiblemente orales o intra-muscular, siempre y cuando no haya contraindicaciones para su uso. Los opioides se deben reservar para casos de dolor agudo y/o severo, debido a sus posibles efectos secundarios como nauseas e íleo.

Alimentación post-operatoria temprana: Al paciente se le debe permitir la ingesta de líquidos claros por vía enteral posterior a la cirugía. De ser posible, el mismo día. Si es tolerada, la ingesta debe ser acompañada de un aumento progresivo de dieta líquida a blanda hasta llegar a una dieta normal en las siguientes 24 horas.

Movilización post-operatoria temprana: Se recomienda desarrollar un plan de movilización ajustado a la edad, estado físico, funcional, y tipo de cirugía, iniciando el mismo día de la cirugía. Este debe ser de carácter progresivo. En casos de pacientes complicados, este plan debe ser ejecutado y guiado de cerca por un(a) fisioterapeuta.

Egreso temprano: Se promueve el egreso temprano del paciente y el regreso a su hogar, con el cuido adecuado de la red de apoyo previamente diseñada y establecida.

ser sometido al estrés quirúrgico que requiere de una valoración pre-quirúrgica exhaustiva y un manejo integral del proceso al que se somete. En este marco, las herramientas mencionadas anteriormente son ampliamente utilizadas en geriatría y cada vez son más utilizadas para la valoración pre-quirúrgica, puesto a que facilitan la valoración inicial y el seguimiento funcional y cognitivo del PAM.

\section{ERAS y el paciente adulto mayor}

El ERAS representa un protocolo seguro para la población geriátrica, en el cual no se han reportado aumento en la readmisión a 30 días posterior a egreso o aumento en la morbimortalidad (23-24). Entre los componentes del ERAS hay unosqueayudan contrarrestar los cambios fi- 


\begin{tabular}{|l|l|}
\hline \multicolumn{1}{|c|}{ Actividades básicas de la vida diaria } & \multicolumn{1}{c|}{ Actividades instrumentales de la vida diaria } \\
\hline Comer & Capacidad de usar el teléfono \\
Bañarse & Elaboración de su propia comida \\
Vestirse y arreglarse & Realizar tareas domésticas básicas \\
Continencia (micción y deposición) & Lavado de su ropa \\
Traslado a sillón-cama & Manejo adecuado de sus medicamentos \\
& Movilidad en la comunidad (uso independiente de me- \\
$\begin{array}{l}\text { Cuadro II: Comparación de actividades de la vida diaria (ABVD) y actividades instrumentales de la vida } \\
\text { diaria (AIVD) }{ }^{(20)} \text {. }\end{array}$
\end{tabular}

siológicos del envejecimiento e impactan al PAM de manera positiva.

Con el envejecimiento, a nivel cardiovascular, hay un aumento gradual de la presión arterial (PA) debido a la disminución de la elasticidad vascular, esto conlleva a una resistencia a la eyección del ventrículo izquierdo lo cual aumenta el trabajo del mismo. Como resultado el miocardio se hace más rígido y resulta en disfunción diastólica la cual está presente en mitad de los PAM ${ }^{(25)}$. La restricción de líquido trans y post quirúrgico previene la recarga del sistema cardiopulmonary la posibilidad de insuficiencia cardiaca congestiva aguda especialmente en el $\mathrm{PAM}^{(26)}$.En las cirugías en que se permite, la anestesia epidural se prefiere sobre la general, esta proporciona un bloqueo aferente neuronal que disminuye la respuesta catabólica común en anestesia regional asimismo disminuyendo el trabajo cardiaco y la posibilidad de descompensaciones cardiacas trans y post quirúrgicas ${ }^{(27)}$.
A nivel del sistema pulmonar, el envejecimiento se acompaña con una disminución de los volúmenes pulmonares debido a la rigidez de la pared torácica, disminución de la elasticidad del parénquima pulmonar y de la respuesta ventilatoria a hipercapnia o hipoxemia ${ }^{(25)}$. En cirugías que aplican anastomosis intestinal, la aplicación de oxígeno a altas concentraciones ayuda al PAM con la oxigenación tisular lo cual aumenta la perfusión en el sitio de anastomosis y disminuye la posibilidad de infección de herida quirúrgica (28). También, reduce posibilidad de hipoxemia la cual es común en el PAM.

Independientemente de la condición basal del PAM, todos se terminan viendo afectados por la hospitalización y el reposo en cama. La falta de movilización y el reposo en cama se combinan para resultar en un declive funcional marcado con des-acondicionamiento funcional y físico ${ }^{(29)}$. Entre las patologías comunes del PAM encamado están; infecciones nosocomiales, úlceras por presión, malnutrición, delirium, trastornos del sueño y caídas ${ }^{(30)}$.

Cuadro III: Características de la fragilidad con base en la definición en el estudio de Fried, L. P. et al.,Estudio de Salud Cardiovascular ${ }^{21}$.

\begin{tabular}{|c|l|}
\hline Característica & \multicolumn{1}{c|}{ Definición } \\
\hline Pérdida de peso & $\begin{array}{l}\text { Pérdida de peso no intencional }>4.5 \mathrm{~kg} \text { o }>5 \% \text { del peso corporal del año ante- } \\
\text { rior. }\end{array}$ \\
\hline Debilidad & $\begin{array}{l}\text { Fuerza de prensión de puño }(\mathrm{kg}) \text { en el quintil más bajo indicado en el Estudio } \\
\text { de Salud Cardiovascular }{ }^{(20)} \text {. (Estratificado por género e índice de masa corpo- } \\
\text { ral (IMC)). }\end{array}$ \\
\hline Cansancio & $\begin{array}{l}\text { Baja resistencia física auto reportada u observada (cuestionario preguntando } \\
\text { con qué frecuencia se siente que toda actividad se realiza con cuanto esfuerzo } \\
\text { o que es difícil iniciar el día) }{ }^{(15)} \text {. }\end{array}$ \\
\hline $\begin{array}{c}\text { Bajo nivel de activi- } \\
\text { dad }\end{array}$ & $\begin{array}{l}\text { Kcal gastadas por semana. Estratificado por género con base en una versión } \\
\text { corta del Cuestionario de Actividades del Tiempo libre de Minnesota }\end{array}$ \\
\hline
\end{tabular}


Cuadro IV: Herramientas para medir el estado funcional del adulto mayor ${ }^{(20)}$.Salud Cardiovascular ${ }^{(21)}$.

\begin{tabular}{|c|c|c|}
\hline Nombre & Contenido & Utilidad principal \\
\hline Índice de Barthel & $\begin{array}{l}\text { Usado para medir la independencia } \\
\text { funcional de las AVD a través de diez } \\
\text { aspectos relacionados al cuido personal } \\
\text { y la movilidad. }\end{array}$ & $\begin{array}{l}\text { Utilizado extensivamente para el moni- } \\
\text { toreo del cambio funcional en pacientes } \\
\text { en recuperación de accidentes cerebro } \\
\text { vasculares. }\end{array}$ \\
\hline $\begin{array}{l}\text { Índice de Capa- } \\
\text { cidad Funcional } \\
\text { (ICF) }\end{array}$ & $\begin{array}{l}\text { Usado para medir la capacidad de reali- } \\
\text { zar AVD, se dividen en diez dimensio- } \\
\text { nes con diferentes niveles de función. } \\
\text { Valora las funciones físicas y cognitivas, } \\
\text { pero no las psicosociales. }\end{array}$ & $\begin{array}{l}\text { Utilizado originalmente en pacientes con } \\
\text { traumas, incluyendo trauma cráneo } \\
\text { encefálico. }\end{array}$ \\
\hline $\begin{array}{l}\text { Medida de Inde- } \\
\text { pendencia Fun- } \\
\text { cional (MIF) }\end{array}$ & $\begin{array}{l}\text { Usado para medir la capacidad funcional } \\
\text { en dos subcategorías: motora y cogni- } \\
\text { ción. Es utilizado en mediciones de } \\
\text { funciones psico-sociales. }\end{array}$ & $\begin{array}{l}\text { Utilizado como complemento del ICF } \\
\text { para detectar cambios durante la rehabi- } \\
\text { litación a corto y mediano plazo. }\end{array}$ \\
\hline Nombre & $\begin{array}{c}\text { Contenido } \\
\end{array}$ & $\begin{array}{r}\text { Utilidad principal } \\
\end{array}$ \\
\hline Índice de Barthel & $\begin{array}{l}\text { Usado para medir la independencia } \\
\text { funcional de las AVD a través de diez } \\
\text { aspectos relacionados al cuido personal } \\
\text { y la movilidad. }\end{array}$ & $\begin{array}{l}\text { Utilizado extensivamente para el moni- } \\
\text { toreo del cambio funcional en pacientes } \\
\text { en recuperación de accidentes cerebro } \\
\text { vasculares. }\end{array}$ \\
\hline $\begin{array}{l}\text { Índice de Capa- } \\
\text { cidad Funcional } \\
\text { (ICF) }\end{array}$ & $\begin{array}{l}\text { Usado para medir la capacidad de reali- } \\
\text { zar AVD, se dividen en diez dimensio- } \\
\text { nes con diferentes niveles de función. } \\
\text { Valora las funciones físicas y cognitivas, } \\
\text { pero no las psicosociales. }\end{array}$ & $\begin{array}{l}\text { Utilizado originalmente en pacientes con } \\
\text { traumas, incluyendo trauma cráneo } \\
\text { encefálico. }\end{array}$ \\
\hline $\begin{array}{l}\text { Medida de Inde- } \\
\text { pendencia Fun- } \\
\text { cional (MIF) }\end{array}$ & $\begin{array}{l}\text { Usado para medir la capacidad funcional } \\
\text { en dos subcategorías: motora y cogni- } \\
\text { ción. Es utilizado en mediciones de } \\
\text { funciones psico-sociales. }\end{array}$ & $\begin{array}{l}\text { Utilizado como complemento del ICF } \\
\text { para detectar cambios durante la rehabi- } \\
\text { litación a corto y mediano plazo. }\end{array}$ \\
\hline
\end{tabular}

La sarcopenia está presente en más de $50 \%$ de los pacientes de la comunidad mayores de 80 años, lo cual es un factor de riesgo para la fragilidad, incapacidad y deterioro rápido del PAM (31). Asimismo, el reposo en cama conlleva a una pérdida de masa muscular de 3 a 6 veces más acelerada en el PAM en comparación al adulto ${ }^{(31)}$. Entre el protocolo ERAS hay varios componentes que promueven la movilización temprana del paciente. El uso de analgésicos no opiodes y el uso conservador de catéteres, sondas y drenos evitan que el paciente tenga restricción a la movilidad o síntomas (dolor, nauseas) que le limiten la ambulación o movilización temprana. De esta manera se promueve la recuperación pronta y egreso temprano.

\section{CONCLUSIÓN}

El protocolo ERAS tiene más de una década de ser utilizado para la población general en diferente tipos de cirugías. Representa un protocolo completo y seguro cuyos diferentes componentes ayudan a disminuir el estrés quirúrgico y mejorar los resultados, particularmente en el PAM.

En el presente y futuro cercano el aumento del cáncer de manejo quirúrgico entre otras cirugías en el PAM es inevitable. Consecuentemente, las necesidades particulares del PAM ante esta situación debe ser tomada en consideración y un aspecto fundamental para ello es el desarrollo de un abordaje integral especializado, en el cual se toman en cuenta las necesidades y tratamiento 
especiales de este grupo etáreo. La valoración y manejo postquirúrgico en el PAM implica invertir el adecuado tiempo y recursos necesarios para que el paciente regrese a su estado funcional, al que se encontraba antes de la intervención.Para que ello sea posible se tiene que garantizar atención adecuada a nivel hospitalario inmediatamente después de la intervención, atendiendo particularmente los aspectos que abarca el programa ERAS, así como el cuido especializado por parte del personal de enfermería y la atención pretendida domiciliar.

\section{BIBLIOGRAFÍA}

1) Rosenthal RA Zenilman ME Katlic MR. The demography of aging. Principles and practice of geriatric surgery. Berlin, Springer. 2012; 2: 138-139.

2) Kehlet, H Wilmore DW. Evidence-Based Surgical Care and the Evolution of FastTrack Surgery. Annals of Surgery. 2008; 248: 189-198.

3) Kehlet, H. Multimodal approach to control postoperative pathophysiology and rehabilitation. British Journal of Anaesthesia, 1997; 78: 606-617.

4) Kehlet, H. Fast-track colorectal surgery. The Lancet. 2008; 371(9615): 791-793

5) Spanjersberg WR Reuring J Keus F van Laarhoven CJHM. Fast track surgery versus conventional recovery strategies for colorectal surgery (review). The Cochrane library. 2011;2:1-47.

6) Aditya J Nanvati S Prabhakar. A comparative study of 'Fast-Track' versus traditional peri-operative care protocols in gastrointestinal surgeries. Journal of gastrointestinal surgery. 2014; 18:757-767.

7) Khan S Gatt M Horgan A et al. Guidelines for implementation of enchanced recovery protocols. Association of Surgeons of Great Britain and Ireland. 2009; 6-16.

8) Thompson EGE Gower ST Beilby DS et al. Enhanced Recovery after surgery program for elective abdominal surgery at three Victorian Hospitals. Anaesthesia and Intensive Care. 2012; 40(3): 450-459.
9) Wilmore DW. Recent advances: Management of patients in fast track surgery. British Medical Journal. 2001; 322(7284): 473-476.

10) Greenfield, L. J., \& Mulholland, M. W. Nutrition and metabolism. In Greenfield's surgery: Scientific principles and practice. Philadelphia, Pa. [u.a.: Lippincott, Williams \& Wilkins. 2011; 5, 65.

11) Kehlet H Wilmore DW. Multimodal strategies to improve surgical outcome. The American Journal of Surgery. 2002; 183(6): 630-641.

12) Faeron KC Ljunggvist $O$ Von Meyenfeldt $\mathrm{M}$ et al. Enhanced recovery after surgery: a consensus review of clinical care for patients undergoing colonic resection. Clinical Nutrition. 2005; 3: 466-477.

13) Basse L Raskov HH Hjort Jakobsen D et al. Accelerated postoperative recovery programme after colonic resection improves physical performance, pulmonary function and body composition. British Journal of Surgery. 2002; 89(4): 446-453.

14) Gatt, M Anderson AD Reddy BS Hayward-Sampson P Tiring, IC MacFie, J. Randomized clinical trial of multimodal optimization of surgical care in patients undergoing major colonic resection. British Journal of Surgery Society. 2005; 92: 1354-1362.

15) Berger NA, Savvides P, Koroukian SM et al. Cancer in the elderly. Transactions of the American clinical and climatological association. 2006; 117, 147-156.

16) Rosenthal RA Zenilman ME Katlic MR. Cancer, carcinogenesis, and aging. Principles and practice of geriatric surgery Berlin, Springer. 2012; 2: 39,46.

17) Berger NA, Savvides P, Koroukian SM et al. Cancer in the elderly. Transactions of the American clinical and climatological association. 2006; 117, 147-156.

18) Kurian AA Wang L Grunkerneier G Bhayani NH Swanström LL. Defining "the elderly" undergoing major gastrointestinal resections: receiver operating characteristic analysis of a large ACS-NSQIP 
cohort. Annals of surgery. 2013; 258: 483489.

19) Biffl WL Biffl SE. Rehabilitation of the geriatric surgical patient: predicting needs and optimizing outcomes. Surgical Clinics of North America. 2015; 95: 173-190.

20) Fried LP Tangen CM Walston J et al. Frailty in Older Adults: Evidence for a Phenotype. The Journals of Gerontology Series A: Biological Sciences and Medical Sciences. 2001; 56: 146-156.

21) Fried LP Borhani NO Enright $P$ et al. The cardiovascular health study: Design and rationale. Annals of Epidemiology, 2001; 3: $263-276$

22) Magaziner J Zimmerman SI GruberBaldini AL Hebel JR Fox KM. Proxy Reporting in Five Areas of Functional Status Comparison with Self-Reports and Observations of Performance. Annals Journal of Epidemiology. 1997; 146(5): 418-28.

23) Thompson EGE Gower ST Beilby DS et al. Enhanced Recovery after surgery program for elective abdominal surgery at three Victorian Hospitals. Anaesthesia and Intensive Care. 2012; 40(3): 450-459.

24) Kurian AA Wang L Grunkerneier G Bhayani NH Swanström LL. Defining "the elderly" undergoing major gastrointestinal resections: receiver operating characteristic analysis of a large ACSNSQIP cohort. Annals of surgery. 2013; 258: 483-489.

25) Barnett S. Anesthesia for the older adult. Up to Date. 2005; topic 14931.

26) Khoo CK Vickery CJ Forsyth $\mathrm{N}$ et al.A prospective randomized controlled trial of multimodal perioperative management protocol in patients undergoing elective colorectal resection for cancer. Ann. Surg. 2007; 245: 867-872.

27) Grigoras I. Fast-Track Surgery, A New Concept, The perioperative Anaesthetic Management. Jurnalul de Chirurgie. 2007; 3: 92-98.

28) Qadan M Akça O Mahid SS et al.Perioperative supplemental oxygen therapy and surgical site infection: a me- ta-analysis of randomized controlled trials. Arch Surg. 2009: 144(4):359-66.

29) Heit JA Silverstein MD Mohr DN et al. Risk factor for Deep vein thrombosis and pulmonary embolism: a population-based case-control study. Archives of Internal Medicine. 2000; 160(60): 809.

30) Mattison M. Hospital management of older adults. 2015; topic 16283.

31) English KL Paddon-Jones D. Protecting muscle mass and function in older adults during bed rest. Current opinion in clinical nutrition \& metabolic care. 2010; 13(1): 34-39.

\section{AGRADECIMIENTOS}

Nuestro agradecimiento va a la Biblioteca Nacional de la Salud y Seguridad Social de la Caja Costarricense de Seguro Social, y la Biblioteca de la UCR por su apoyo y paciencia en la búsqueda de los artículos que fueron usados para la elaboración de esta revisión.

\section{FUENTES DE APOYO}

No hay fuentes de apoyo que declarar.

\section{CONFLICTO DE INTERÉS Y/O AGRADE- CIMIENTOS}

Los autores declaran que no existió ningún conflicto de interés en el presente reporte. 